Annals of Pure and Applied Mathematics

Vol. 16, No. 2, 2018, 461-469

ISSN: 2279-087X (P), 2279-0888(online)

Published on 27 March 2018

www.researchmathsci.org

DOI: http://dx.doi.org/10.22457/apam.v16n2a24

Annals of

Pure and Applied

Mathematics

\title{
Perfectly Regular and Perfectly Edge-regular Fuzzy Graphs
}

\section{Michael Cary}

Center for Alternative Fuels, Engines, and Emissions, West Virginia University

Morgantown, WV, USA. E-mail: macary@ mail.wvu.edu

Received 17 January 2018; accepted 16 February 2018

Abstract. A perfectly regular fuzzy graph is a fuzzy graph that is both regular and totally regular. A perfectly edge-regular fuzzy graph is a fuzzy graph that is both edge-regular and totally edge-regular. In this paper, we introduce and classify these types of fuzzy graphs and study several of their properties, including how these two classes of fuzzy graphs structurally relate to one another and several of their spectral properties such as isospectral fuzzy graphs and when the energy of fuzzy graphs is proportional to the energy of their underlying crisp graphs. These properties are studied in particular due to their having at least one constant function between $\mu$ and $\sigma$.

Keywords: Fuzzy graph, perfectly regular, perfectly edge-regular, graph energy, spectral fuzzy graph theory, fuzzy matrix

AMS Mathematics Subject Classification (2010): 05C72, $03 E 72$

\section{Introduction}

Regular and totally regular fuzzy graphs were first introduced in [5]. The fuzzy edge analog of these concepts, edge-regularity and total edge-regularity, were introduced and studied in [18]. These notions of regularity were shown to be analogous to regularity in the standard graph theoretic context. These concepts of regularity for both vertices and edges in fuzzy graphs led to many advancements in the structural theory of fuzzy graphs. Several relevant marquee results stemming from this research include [1,3,4,6,8-17,19-23,25-30].

The purpose of this paper is to prepare for a study those fuzzy graphs that concurrently exhibit both fuzzy vertex and edge-regular properties. These graphs will eventually help link certain fuzzy systems and crisp systems, allowing for greater ease in computing properties of these fuzzy systems for modeling pruposes [2] or optimizing these fuzzy networks [7]. We first study perfectly regular fuzzy graphs, i.e. those fuzzy graphs which are both regular and totally regular, to find necessary conditions for vertex regularity in moderately crisp graphs. We then study perfectly edge-regular fuzzy graphs, i.e. those fuzzy graphs which are both edge-regular and totally edge-regular. Spectral properties of these classes of fuzzy graphs in particular will help relate these notions of regularity in fuzzy graphs to crisp graphs, thus allowing for a deeper understanding of these special classes of fuzzy graphs.

Perfectly regular fuzzy graphs will be characterized in Section 2 along with several initial results on perfectly regular fuzzy graphs. A similar study of perfectly edge-regular 


\section{Michael Cary}

fuzzy graphs will be given in Section 3. From there, we will study the combination of these properties in fuzzy graphs by first studying the relationships between perfectly regular and perfectly edge-regular fuzzy graphs in Section 4 and then by providing a study of their adjacency matrices in Section 5. The intention of this body of work is to serve as the necessary preliminaries for the introduction and study of those fuzzy graphs exhibiting concurrently constant functions $\mu$ and $\sigma$, including those fuzzy graphs which are both perfectly regular and perfectly edge-regular. Before we proceed, however, a few relevant definitions are necessary. Throughout this paper we define the vertex set $V$ of a fuzzy graph to satisfy $\sigma(v)>0$ for all $v \in V$, i.e. the vertex set of the underlying graph of a fuzzy graph is the same as the vertex set of the fuzzy graph itself, i.e. the fuzzy graph contains no necessarily isolated vertices. Edges are defined similarly. For an introduction to fuzzy graph theory and its basic definitions, the reader is referred to [31]. For analysis notations and relevant limit theorems, the reader is referred to [24].

Definition 1. The degree of a vertex in a fuzzy graph is $d(v)=\sum_{u \neq v} \mu(v u)$.

Definition 2. The total degree of a vertex in a fuzzy graph is $t d(v)=d(v)+\sigma(v)$.

Definition 3. A regular fuzzy graph is a fuzzy graph with $d(v)=k \forall v \in V$.

Definition 4. A totally regular fuzzy graph is a (not necessarily regular) fuzzy graph with $t d(v)=k \forall v \in V$.

Definition 5. A perfectly regular fuzzy graph is a fuzzy graph that is both regular and totally regular.

Definition 6. The degree of an edge in a fuzzy graph is $d(u v)=d(u)+d(v)-2 \mu(u v)$.

Definition 7. The total degree of an edge in a fuzzy graph is $t d(u v)=d(u v)+\mu(u v)$.

Definition 8. An edge-regular fuzzy graph is a fuzzy graph having $d(u v)=k \forall u v \in E$.

Definition 9. A totally edge-regular fuzzy graph is a fuzzy graph with $t d(u v)=k \forall u v \in$ E.

Definition 10. A perfectly edge-regular fuzzy graph is a fuzzy graph that is both edge-regular and totally edge-regular.

Definition 11. The order of a fuzzy graph is $O(\xi)=\sum_{v \in V} \sigma(v)$.

Definition 12. The size of a fuzzy graph is $S(\xi)=\sum_{u v \in E} \mu(u v)$.

Definition 13. A fuzzy graph is complete if $\mu(u v)=\sigma(u) \wedge \sigma(v) \forall u v \in E$.

\section{Perfectly regular fuzzy graphs}

Let $\xi=(V, \sigma, \mu)$ be a fuzzy graph. In [5], examples were provided of regular fuzzy graphs 


\section{Perfectly Regular and Perfectly Edge-regular Fuzzy Graphs}

that are not totally regular and of totally regular fuzzy graphs that are not regular. In that same work, Theorem 2.11 provided a necessary (but not sufficient) condition on the fuzzy subset $\sigma$ of $V$ for perfectly regular graphs, namely that $\sigma: V \rightarrow[0,1]$ must be a constant function. We present a modified version of that proof as it fits the context of perfectly regular fuzzy graphs now for completeness.

Lemma 1. Let $\xi=(V, \sigma, \mu)$ be a perfectly regular fuzzy graph. Then $\sigma: V \rightarrow[0,1]$ is a constant function.

Proof: Since $\xi$ is perfectly regular, we have that $\xi$ is both $k_{1}$-regular and $k_{2}$-totally regular. Then we have that $t d(v)=d(v)+\sigma(v)=d(u)+\sigma(u)=t d(u) \forall u, v \in V$. Since $d(v)=d(u)=k_{1}$ and $t d(v)=t d(u)=k_{2}$, we have that $\sigma(v)=\sigma(u)$, hence if $\xi$ is perfectly regular, then $\sigma$ must be a constant function.

However, it is obvious that this is not a sufficient condition, as any irregular fuzzy graph with a constant function $\sigma$ will be neither totally nor perfectly regular. Thus we may classify perfectly regular fuzzy graphs as precisely the graphs that are regular with a constant $\sigma$.

Theorem 1. A fuzzy graph $\xi=(V, \sigma, \mu)$ is perfectly regular if and only if it satisfies the following conditions:

$$
\begin{aligned}
\text { (i) } \sum_{k \neq i} \mu\left(v_{i} v_{k}\right) & =\sum_{k \neq j} \mu\left(v_{j} v_{k}\right) \forall i, j \in\{1, \ldots,|V|\} \\
\text { (ii) } \sigma\left(v_{i}\right) & =\sigma\left(v_{j}\right) \forall i, j \in\{1, \ldots,|V|\}
\end{aligned}
$$

Proof: $(\Rightarrow)$ Let $\xi$ be perfectly regular. By definition $\xi$ is regular, hence it trivially satisfies $(i)$. Lemma 1 implies that condition (ii) is also met.

$(<=)$ Let $\xi$ be a fuzzy graph satisfying both conditions $(i)$ and (ii). Since $(i)$ is the definition of regularity, $\xi$ is $k$-regular. Since by (ii) we have that $\sigma=c$ is a constant function, $t d(v)=k+c \forall v \in V$ and thus $\xi$ is both regular and totally regular, hence $\xi$ is perfectly regular.

This theorem provides a comprehensive classification of perfectly regular fuzzy graphs as precisely those fuzzy graphs that are regular with constant $\sigma$. With this definition now well established, we are ready to prove some initial properties of perfectly regular fuzzy graphs.

Observation 1. Let $\xi$ be a perfectly regular fuzzy graph and let $\sigma(v)=c \forall v \in V$. Then the order of $\xi$ is $O(\xi)=c|V|$.

Proposition 1. Let $\xi$ be a perfectly regular fuzzy graph and let $d(v)=k \forall v \in V$. Then the size of $\xi$ is $S(\xi)=\frac{k|V|}{2}$.

Proof: Since $\xi$ is perfectly regular, we have that $d(v)=k \forall v \in V$. Hence $\sum_{v \in V} d(v)=k|V|$. However, since $d(v)=\sum_{u \neq v} \mu(u v)$, we have that $\sum_{v \in V} d(v)=$ $\sum_{v \in V} \sum_{u \neq v} \mu(u v)=2 \sum_{u v \in E} \mu(u v)=2 S(\xi)$. Thus we conclude that the size of a perfectly regular fuzzy graph is $\frac{k|V|}{2}$.

\section{Perfectly edge-regular fuzzy graphs}

Edge-regularity was pioneered in [3], and therein it was shown that the edge-regularity property is a strong analog of regularity in fuzzy graphs. In an effort to help solidify the 


\section{Michael Cary}

definition of moderately crisp graphs, we will demonstrate results on perfect edge-regularity analogous to those given in Section 2 on perfect regularity in fuzzy graphs.

Lemma 2. Let $\xi=(V, \sigma, \mu)$ be a perfectly edge-regular fuzzy graph. Then $\mu: V \times V \rightarrow$ $[0,1]$ is a constant function.

Proof: Since $\xi$ is perfectly edge-regular, we have that $\xi$ is both $k_{1}$-edge-regular and $k_{2}$-totally edge-regular. Then we have that $k_{2}=t d(u v)=d(u v)+\mu(u v)=k_{1}+$ $\mu(u v)=t d(x y)=d(x y)+\mu(x y)=k_{1}+\mu(x y)$ hence $\mu(u v)=\mu(x y)$. Since $u v$ and $x y$ were arbitrarily chosen edges, the proof is complete.

As in the previous section pertaining to vertices, this does not constitute a sufficient condition for a fuzzy graph to be perfectly edge-regular. We classify the perfectly edge-regular fuzzy graphs with the following theorem.

Theorem 2. A fuzzy graph $\xi=(V, \sigma, \mu)$ is perfectly edge-regular if and only if it satisfies the following conditions:

(i) $\sum_{z \neq u} \mu(u z)+\sum_{z \neq v} \mu(v z)-2 \mu(u v)=\sum_{z \neq x} \mu(x z)+\sum_{z \neq y} \mu(y z)-2 \mu(x y) \forall u v, x y$

$$
\in E
$$

(ii) $\mu(u v)=\mu(x y) \forall u v, x y \in E$.

Proof: $(\Rightarrow)$ Since $\xi$ is perfectly edge-regular and since $(i)$ is the definition of an edge-regular fuzzy graph, $\xi$ obviously satisfies $(i)$. By Lemma 2 , $\xi$ also satisfies (ii).

$(<=)$ Let $\xi$ be a fuzzy graph satisfying both $(i)$ and (ii), i.e. $\xi$ is $k$-edge-regular and has a constant $\mu=c$. Since ( $i$ ) is the definition of being edge-regular, (ii) implies that $t d(u v)=k+c=t d(x y) \forall u v, x y \in E$.

Thus we see that the perfectly edge-regular fuzzy graphs are precisely those edge-regular fuzzy graphs with constant $\mu$, a nice analog to the case considering vertices of fuzzy graphs in the previous section. We next provide some results on the order and size of perfectly edge-regular graphs.

Observation 2. Let $\xi$ be a perfectly edge-regular fuzzy graph and let $\mu(u v)=c \forall u v \in$ $E$. Then the size of $\xi$ is $S(\xi)=c|E|$.

This is the edge-analog of the vertex implication on the order of a regular fuzzy graph.

Next we consider the order of a perfectly edge-regular fuzzy graph. Unlike the problem of determining the size of a perfectly regular fuzzy graph in the previous section, we can no longer provide an explicit general formula for the order of a perfectly edge-regular fuzzy graph in the absence of further assumptions. We can, however, bound the order of a perfectly edge-regular fuzzy graph.

Theorem 3. Let $\xi$ be a perfectly edge-regular fuzzy graph. Then the order of $\xi$ is bounded between $\sum_{v \in V} \vee_{x \neq v} \mu(x v) \leq O(\xi) \leq|V|$.

Proof: As the upper bound is obvious, we need only to prove the lower bound. This follows directly from the definition of $\mu(u v) \leq \sigma(u) \wedge \sigma(v)$. Thus we have that $\sigma(v) \geq \mathrm{V}_{x \neq v} \mu(x v)$ is a lower bound for $\sigma(v) \forall v \in V$. The sum of these individual lower bounds for $\sigma(v)$ is precisely the lower bound stated for $O(\xi)$ in the theorem.

We note that a more concise (in terms of parameters) though slightly worse lower bound would be $|V| \cdot(\wedge \vee \mu(u v))$. 


\section{Perfectly Regular and Perfectly Edge-regular Fuzzy Graphs}

One might expect that, in the presence of more information on the perfectly edge-regular fuzzy system $\xi$, one could given an explicit formula for $O(\xi)$. However, even when $\xi$ is complete, we find that this is not always possible. In some special cases we may determine the exact value of $O(\xi)$ this way, but, in general, what we can obtain regarding the order of a perfectly-edge regular complete fuzzy graph is a result on the use of the lower bound presented above as an approximation for $O(\xi)$. These two results are presented in the ensuing theorems and use the immediately following lemma.

Lemma 3. Let $\xi$ be a perfectly edge-regular complete fuzzy graph and let $u \in V$. If $\exists v \in N(u)$ such that $\sigma(u) \leq \sigma(v)$, then there exists an edge uv $\in E$ such that $\mu(u v)=\sigma(u)$.

Proof: Since $\xi$ is complete, $\mu(u v)=\sigma(u) \wedge \sigma(v)$. Choose $v$ such that $v \in N(u)$ and $\sigma(v) \geq \sigma(u)$. Then $\mu(u v)=\sigma(u)$.

Lemma 4. Let $\mathcal{P}_{u}$ be an ordering of the $v \in N(u)$ such that $x \leq y=>\sigma(x) \leq \sigma(y)$. If $\mathcal{P}_{u}$ does not have a unique greatest element for all $u \in V$, then $\sum_{v \in V} \wedge_{x \neq v} \mu(x v)=$ $O(\xi)$.

Proof: Since $\xi$ is complete and since there is no unique greatest element in $\mathcal{P}_{u}$ for all $u \in V$, we have that $\forall v \in V \exists u \in V$ s.t. $\sigma(v) \leq \sigma(u)$. By Lemma 3, we have that $\sum_{v \in V} \wedge_{x \neq v} \mu(x v)=O(\xi)$.

The above lemma shows that, in a special case, we can directly compute the order of a perfectly edge-regular complete fuzzy graph. However, this is not the case in general. From the previous lemma, one may guess that $\sum_{v \in V} \wedge_{x \neq v} \mu(x v)=c|V|$ is a decent approximation for the order of a perfectly edge-regular complete fuzzy graph. We use the following theorem to strengthen the validity of this sum as an approximation of the order of a perfectly edge-regular complete fuzzy graph.

Theorem 4. Let $O(\xi)$ represent the order of a perfectly edge-regular complete fuzzy graph $\xi$ with constant $\mu=c$, let $\mathcal{J}$ be the independent set of all vertices $v$ such that $\nexists u v \in E$ such that $\mu(u v)=\sigma(v)$, and let $|\mathcal{J}|=\alpha|V|$. Then $(1-\alpha(1-c)) O(\xi) \leq$ $\sum_{v \in V} \wedge_{x \neq v} \mu(x v) \leq O(\xi)$.

Proof: As the upper bound was established in Theorem 3, we need only to prove the lower bound. Let $V_{1}$ be the set of vertices $v \in V$ such that $\exists u v \in E$ such that $\mu(u v)=\sigma(v)$, and let $V_{2}=V \backslash V_{1}$, i.e. $V_{2}$ is the independent set described in the statement of the theorem, hence $\left|V_{2}\right|=\alpha|V|$. Thus we may write a lower bound for $O(\xi)$ as

$\sum_{v \in V_{1}} \wedge_{x \neq v} \mu(x v)+\sum_{v \in V_{2}} \wedge_{x \neq v} \mu(x v) \leq \sum_{v \in V_{1}} \sigma(v)+\sum_{v \in V_{2}} \sigma(v)$

By definition of $V_{1}$ we have that $\sum_{v \in V_{1}} \wedge_{x \neq v} \mu(x v)=\sum_{v \in V_{1}} \sigma(v)$, so we may rewrite this lower bound as $\sum_{v \in V_{2}} \wedge_{x \neq v} \mu(x v) \leq \sum_{v \in V_{2}} \sigma(v)$ with equality holding only when $V_{2}=\emptyset$. Since $\mu=c$ is a constant function due to the perfect edge-regularity of $\xi$, we may obtain an upper bound on the difference between the two sides of our inequality as

$$
\alpha(1-c)|V| \geq \sum_{v \in V_{2}} \sigma(v)-\sum_{v \in V_{2}} \wedge_{x \neq v} \mu(x v)
$$

Thus we may bound $\sum_{v \in V} \wedge_{x \neq v} \mu(x v)$ in both directions as follows

$$
\sum_{v \in V} \sigma(v)-\alpha(1-c)|V| \leq \sum_{v \in V} \wedge_{x \neq v} \mu(x v) \leq \sum_{v \in V} \sigma(v)
$$

By dividing through by $O(\xi)$ we obtain

$$
1-\frac{\alpha(1-c)|V|}{O(\xi)} \leq \frac{\sum_{v \in V} \wedge_{x \neq v} \mu(x v)}{O(\xi)} \leq 1
$$




\section{Michael Cary}

By noticing that $1 \leq \frac{|V|}{O(\xi)} \leq \frac{1}{c}$ and making a small sacrifice to our lower bound, we obtain

$$
1-\alpha(1-c) \leq \frac{\sum_{v \in V} \wedge_{x \neq v} \mu(x v)}{O(\xi)} \leq 1
$$

By multiplying through by $O(\xi)$ we complete the proof.

Thus we establish what is a decent estimator of the order of an edge-regular fuzzy graph. In particular, this result generalizes Lemma 4 since this would amount to the case where $\alpha=0$ and the lower and upper bounds become equal.

\section{Relating vertex and edge-regularity in fuzzy graphs}

In this section, we study what additional properties are needed for one form of regularity in fuzzy graphs to imply the other form of regularity. As we saw in Theorem 1, a perfectly regular fuzzy graph is a fuzzy graph that is regular and has a constant function $\sigma$. The first question we ask relating regular and edge-regular fuzzy graphs is: What happens if a (perfectly/totally) regular fuzzy graph has a constant function $\mu$ ?

Lemma 5. If $\xi$ is regular and $\mu=c$ is a constant function then $\xi$ is perfectly edge-regular.

Proof: Let $\xi$ be a regular fuzzy graph and let $\mu=c$ be a constant function. Then $d(v)=k \forall v \in V$ where $k$ is simply a multiple of $c$. The degree of an arbitrary edge of $\xi$ is then $d(u v)=d(u)+d(v)-2 \mu(u v)=2 k-2 c=2(k-c)$ hence $\xi$ is edge-regular. Since the total degree of an edge in a fuzzy graph is $t d(u v)=d(u v)+$ $\mu(u v)$, we have that $t d(u v)=2(k-c)+c=2 k-c$ for all edges in $\xi$. Thus $\xi$ is totally edge-regular and therefore perfectly edge-regular.

Since perfectly regular fuzzy graphs are regular, perfectly regular fuzzy graphs with a constant function $\mu$ are necessarily perfectly edge-regular. However, it is not the case in general that totally regular fuzzy graphs with a constant function $\mu$ are perfectly edge-regular; just considerthe following counterexample (Figure 1).

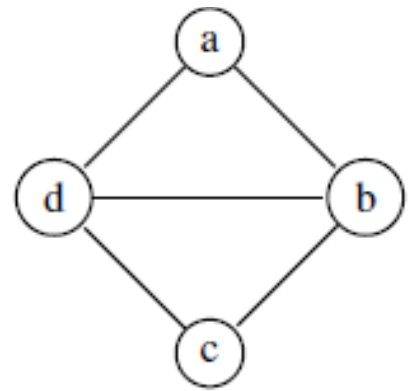

Figure 1:

Let $\sigma(a)=\sigma(c)=0.2$, let $\sigma(b)=\sigma(d)=0.1$, and let $\mu=.1$ be a constant function. Then the degree of $a$ and $c$ is 0.2 while the degree of $b$ and $d$ is 0.3 , yet the total degree of every vertex is precisely 0.4 . Hence this counterexample has a constant $\mu$ and is totally regular, but is not regular. To see that it is not edge-regular, consider the edges $a b$ and $b d$. We have that $d(a b)=0.3 \neq 0.4=d(b d)$. Since $\mu$ is constant, clearly this counterexample cannot be totally edge-regular as it is not edge-regular. 


\section{Perfectly Regular and Perfectly Edge-regular Fuzzy Graphs}

Theorem 5. If $\xi$ is perfectly regular and complete then $\xi$ is perfectly edge-regular. Proof: Since $\xi$ is perfectly regular, $\sigma(u)=\sigma(v) \forall u, v \in V$. Since $\xi$ is complete, $\mu(u v)=\sigma(u) \wedge \sigma(v) \forall u v \in E$. Combined, these two facts yield that $\mu$ is a constant function. Thus we may apply Lemma 5 and complete the proof.

\section{Some spectral properties of edge-regular fuzzy graphs}

In this section, we study some basic spectral properties of perfectly edge-regular fuzzy graphs. The adjacency matrix of a fuzzy graph, $A(\xi)$, is defined as $A(\xi)=\left(a_{i j}\right)=$ $\mu\left(v_{i} v_{j}\right)$. Given this, we will focus our attention on the perfectly edge-regular fuzzy graphs since these graphs satisfy the property that $\mu$ is a constant function. Accordingly, we begin with the following observation.

Observation 3. Let $\xi$ be a perfectly edge-regular fuzzy graph with $\mu=c$ and let $G$ be the underlying crisp graph of $\xi$. Then $A(\xi)=c A(G)$.

Given this simple observation, we can immediately enumerate several spectral properties of perfectly edge-regular fuzzy graphs.

Theorem 6. Let $\xi$ be a perfectly edge-regular fuzzy graph and let $G$ be its underlying crisp graph. If $\lambda$ is an eigenvalue of $G$ then $c \lambda$ is an eigenvalue of $\xi$.

Proof: This follows directly from the fact that $A X=\lambda X$ implies $c A X=c \lambda X$.

Theorem 7. Let $\xi_{1}$ and $\xi_{2}$ be two perfectly edge-regular fuzzy graphs with respective underlying crisp graphs $G_{1}$ and $G_{2}$, respectively. If $G_{1}$ and $G_{2}$ are isospectral and $c_{1}=c_{2}$, then $\xi_{1}$ and $\xi_{2}$ are isospectral.

Proof: From Theorem 6 we have that the multiplicities of the eigenvalues will be unchanged and that the eigenvalues of the fuzzy graphs will both be scaled by $c$, hence $\xi_{1}$ and $\xi_{2}$ are isospectral.

Theorem 8. Let $\xi$ be a perfectly edge-regular fuzzy graph and let $G$ be its underlying crisp graph. If the energy of $G$ is $E(G)$, then the energy of $\xi$ is $E(\xi)=c E(G)$.

Proof: By definition the energy of $G$ is given by $E(G)=\sum_{i=1}^{n}\left|\lambda_{i}\right|$ where the $\lambda_{i}$ are the eigenvalues of $G$. By Theorem 6 we have that the energy of $\xi$ is given by $E(\xi)=$ $\sum_{i=1}^{n}\left|c \lambda_{i}\right|=c \sum_{i=1}^{n}\left|\lambda_{i}\right|=c E(G)$.

\section{Conclusion}

In this paper, we began a systematic study of two classes of fuzzy graphs, perfectly regular and perfectly edge-regular fuzzy graphs, that link fuzzy graph theory to graph theory in several important aspects, most notably by studying some of the structural and spectral properties of these graphs. Given the ubiquity of graph theory in applied settings and the growing implementation of fuzzy graph theory in applied settings, linking together these two types of graphs offers a potential means to gain the extra benefits of a generalized or more flexible model while restricting the computational effort needed to gain these benefits.

Acknowledgements. The author would like to thank the anonymous referees for their insight and suggestions. 


\section{Michael Cary}

\section{REFERENCES}

1. M.Akram and W.A.Dudek, Regular bipolar fuzzy graphs, Neural Computing and Applications, 21(1) (2012) 197-205.

2. S Elizabeth and L Sujatha, Medical diagnosis based on interval valued fuzzy number matrices, Annals of Pure and Applied Mathematics, 7(1) (2014) 91-96.

3. A.Nagoor Gani and M.Basheer Ahamed, Order and size in fuzzy graphs, Bulletin of Pure and Applied Sciences, 22(1) (2003) 145-148.

4. A.Nagoor Gani and S.R.Latha, On irregular fuzzy graphs, Applied Mathematical Sciences, 6(11) (2012) 517-523.

5. A.Nagoor Gani and K.Radha, On regular fuzzy graphs, Journal of Physical Sciences, 12 (2008) 33-40.

6. A.Kishore and M.S.Sunitha, Strong chromatic number of fuzzy graphs, Annals of Pure and Applied Mathematics, 7(2) (2014) 52-60.

7. L'aszl 'o T K'oczy, Fuzzy graphs in the evaluation and optimization of networks, Fuzzy Sets and Systems, 46(3) (1992) 307-319.

8. N.R.Santhi Maheswari and C.Sekar, On $m$-neighbourly irregular fuzzy graphs, International Journal of Mathematics and Soft Computing, 5(2) (2015) 145-153.

9. N.R.Santhi Maheswari and C.Sekar, On $(m, k)$-regular fuzzy graphs, International Journal of Mathematical Archive, 7(1) (2016) 1-7.

10. N.R.Santhi Maheswari and C.Sekar, On pseudo regular fuzzy graphs, Annals of Pure and Applied Mathematics, 11(1) (2016) 105-113.

11. S.Maheswari and C.Sekar, On (2, k)-regular and totally $(2, \mathrm{k})$-regular fuzzy graphs, International Journal of Mathematics and Soft Computing, 4(2) (2014) 59-69.

12. J.Manora and I.Paulraj Jayasimman, Neighborhood sets and neighborhood polynomial of cycles, Annals of Pure and Applied Mathematics, 7(2) (2014) 45-51.

13. S.P.Nandhini and E.Nandhini, Strongly irregular fuzzy graphs, International Journal of Mathematical Archive, 5(5) (2014)110-114.

14. S.Ravi Narayanan and N.R.Santhi Maheswari, Highly totally irregular fuzzy graphs, Journal of Computer and Mathematical Sciences, 4(6) (2013) 403-459.

15. M.Pal and H.Rashmanlou, Irregular interval-valued fuzzy graphs, Annals of Pure and Applied Mathematics, 3(1) (2013) 56-66.

16. K.Radha and N.Kumaravel, On edge regular fuzzy graphs, International Journal of Mathematical Archive, 5(9) (2014) 100-112.

17. K.Radha and N.Kumaravel, On edge regular bipolar fuzzy graphs, Annals of Pure and Applied Mathematics, 10(2) (2015) 129-139.

18. K.Radha and N.Kumaravel, The edge degree and the edge regular properties of truncations of fuzzy graphs, Bulletin of Mathematics and Statistics Research, 4(3) (2016) 7-16.

19. K.Radha and N.Kumaravel, On edge regular square fuzzy graphs, International Journal of Applied Mathematical Sciences, 9(2) (2016) 169-176.

20. K.Radha and M.Vijaya, Totally regular property of the join of two fuzzy graphs, International Journal of Fuzzy Mathematical Archive, 8(1) (2015) 9-17.

21. P.Rajarajeswari and P.Dhanalakshmi, Interval valued fuzzy soft matrix theory, Annals of Pure and Applied Mathematics, 7(2) (2014) 61-72.

22. S.Samanta and M.Pal, Irregular bipolar fuzzy graphs, International Journal of Applications of Fuzzy Sets, 2 (2012) 91-102. 
Perfectly Regular and Perfectly Edge-regular Fuzzy Graphs

23. S.Samanta, B.Sarkar, D.Shin and M.Pal, Completeness and regularity of generalized fuzzy graphs, SpringerPlus, 5(1) (2016) 1979.

24. S.Samanta and M.Pal, Fuzzy $k$-competition graphs and $p$-competition fuzzy graphs, Fuzzy Inf. Eng., 5(2) (2013) 191-204.

25. A.Edward Samuel and C.Kayalvizhi, On $k$-regular chromatic fuzzy graph, Advances in Fuzzy Sets and Systems, 19(2) (2015) 155.

26. N.R.Santhi Maheswari and C.Sekar, On $(r, m, k)$-regular fuzzy graphs, Mathematical Combinatorics, 1 (2016) 18-26.

27. N.R.Santhimaheswari and C.Sekar, On strongly edge irregular fuzzy graphs, Kragujevac Journal of Mathematics, 40(1) (2016) 125-135.

28. S.Sebastian and Ann Mary Philip, Regular and edge regular interval valued fuzzy graphs, Journal of Computer and Mathematical Sciences, 8(7) (2017) 309-322.

29. M.S.Sunitha and S.Mathew, Fuzzy graph theory: a survey, Annals of Pure and Applied Mathematics, 4(1) (2013) 92-110. 